\title{
Processus de Décentralisation au Mali et Couches Sociales Marginalisées
}

\section{Bréhima Béridogo}

\section{Q OpenEdition}

\section{Journals}

Édition électronique

URL : http://journals.openedition.org/apad/581

DOI : 10.4000/apad.581

ISSN : 1950-6929

Éditeur

LIT Verlag

\section{Édition imprimée}

Date de publication : 1 décembre 1997

\section{Référence électronique}

Bréhima Béridogo, "Processus de Décentralisation au Mali et Couches Sociales Marginalisées », Bulletin de l'APAD [En ligne], 14 | 1997, mis en ligne le 26 janvier 2007, consulté le 08 septembre 2020. URL : http://journals.openedition.org/apad/581; DOI : https://doi.org/10.4000/apad.581

Ce document a été généré automatiquement le 8 septembre 2020

Bulletin de I'APAD 


\title{
Processus de Décentralisation au Mali et Couches Sociales Marginalisées
}

\author{
Bréhima Béridogo
}

1 Le Mali, en 1960, hérite de la colonisation un Etat jacobin très centralisé. L'organigramme de son administration est le suivant : à la base 205 arrondissements se répartissent entre 52 cercles. Ces derniers à leur tour sont répartis entre 9 régions. Bamako a un statut particulier, celui d'un district composé de 6 communes. 13 autres villes de l'intérieur ont le statut de commune. Avec la décentralisation, les arrondissements doivent disparaître et laisser la place à 702 communes. Ces différentes structures ont, respectivement, comme administrateur: le chef d'arrondissement, le commandant de cercle, le gouverneur de région ou de district et le maire.

2 Mais comme pendant l'ère coloniale, cette administration a, comme cheville ouvrière de son système, le "commandant de cercle". A son sujet, Jean Suret Canale dit qu'il "administre, perçoit l'impôt, rend la justice, fait la police, gère les prisons, dirige tous les services publics..." (Suret Canale 1977: 95). Avec la déconcentration de l'administration à partir de 1960, le chef d'arrondissement a été amené à jouer le même rôle et mieux, à faire office de police judiciaire. C'est une administration de commandement au sens propre du terme et demeurée comme telle même quand la deuxième République déclara l'avoir transformée en "administration de développement". D'ailleurs, on peut se demander si le régime de l'indigénat, qui donnait aux autorités administratives le droit de frapper de sanctions pénales les administrés sans en référer ou en justifier devant aucune autorité judiciaire, a jamais été aboli dans la pratique. En effet, les commandants de cercle continuent à sévir, emprisonnant et soumettant au bain de soleil en pleine période de canicule les chefs de lignage qui n'arrivent pas à s'acquitter des diverses taxes. L'administration malienne d'avant mars 91 a obligé les populations à adhérer à des organisations et associations (parti unique, coopérative, association de parents d'élève...) et à leur faire payer, en conséquence, des cotisations contre leur gré. 
3 Il s'agit, en principe, pour la décentralisation de corriger cette situation. Amener les populations d'une "stratégie d'observation passive" (Félix 1996: 152) de groupes subissant les contraintes et exactions administratives à une stratégie participative d'acteurs conscients de leur développement.

4 Cette décentralisation, même si elle avait été, théoriquement, initiée et voulue depuis l'indépendance, sa mise en œuvre demeure aujourd'hui la suite logique du changement politique intervenu depuis mars 1991 au Mali. Elle sera aussi, mise en œuvre dans un cadre démocratique, c'est-à-dire dans un contexte de pluralisme politique ce qui fait une différence fondamentale entre la situation présente et les intentions des Etats post-coloniaux et les pratiques des Etats pré-coloniaux dont se prévalent les "fondamentalistes culturels" du Mali. Ceux-ci, en effet, prétendent que la décentralisation n'est pas un phénomène étranger au Mali, mais qu'elle était la forme d'administration des Etats pré-coloniaux (empire du Mali, du Songhoy, royaume de Ségou...).

5 Les soulèvements de 1990-1991 ayant surtout eu lieu en milieu urbain et ayant été dirigés par les intellectuels, la décentralisation, selon ses initiateurs, devrait permettre l'attelage du milieu rural à la locomotive de la démocratie. Elle devrait offrir un espace d'expression au monde rural, contribuant ainsi à l'ancrage de la démocratie au village.

6 Mais le monde rural fonctionne-t-il traditionnellement de manière démocratique? Fera-t-il de la décentralisation la même lecture que les initiateurs du phénomène, l'Etat et ses intellectuels ? Comment la mise en œuvre du processus s'est-elle faite jusqu'ici? Comment l'information a-t-elle circulé et comment les décisions ont-elles été prises? Toutes les couches ont-elles été associées? Voilà tout un ensemble de questionnement auxquels le présent article tentera de répondre.

Les structures sociales villageoises

7 Dans la plupart des groupes ethniques maliens (Bambara, Malinké, Soninké, Bozo, Sénoufo, Minyanka, Bwa, Dogon, Peul, Songhay, Touareg, Maure...) il existe différents types de structures; certaines basées sur l'âge, d'autres sur la division en castes. Certains groupes, cependant, comme les Sénoufo et les Minyanka ignorent l'existence des castes ${ }^{1}$.

8 La séniorité, conférée par l'âge biologique, est régie par la préséance dans la naissance, la primogéniture. Ce statut d'aîné entraîne le droit d'exercer un pouvoir sur les cadets. Ainsi, sous un certain angle, la structure sociale est déterminée par les types de rapports liant aînés et cadets. Dans le cadre de l'administration villageoise, les aînés sont les chefs de lignage, de famille (groupe domestique de production et de reproduction) et les adultes initiés faisant généralement partie de la génération des pères d'un lignage.

9 L'âge social, fondé sur le statut dans le cadre de la parenté, confère la séniorité, le statut d'aîné à une personne, même si celle-ci est biologiquement moins âgée. Il fait ainsi des femmes d'éternels cadets. Elles vont s'ajouter aux jeunes pour former le groupe des cadets (Meillassoux 1975 : 116-121).

10 La séniorité est synonyme de détention de pouvoir et de savoir. Dans ces domaines, l'aîné a toujours un plus par rapport au cadet. Au niveau villageois, les aînés sont les premiers responsables de l'administration des hommes et des biens. Les cadets, jeunes et femmes, sont beaucoup plus des exécutants et surtout plus interpellés et présents dans le cadre de la production. 
11 Parallèlement, il existe une autre division sociale. C'est celle qui divise la société en une caste supérieure et des castes inférieures composées des artisans et des artistes. L'appartenance à une caste confère un statut social auquel sont liés des attributs. Ainsi, chez les Mandingues, les quatre principaux attributs du hàron (homme de la caste supérieure) sont : la vérité (il ne ment jamais), le respect de la parole donnée (il ne se dédit jamais, ne trahit jamais), le courage (il se nourrit de l'agriculture ou du commerce et ne fuit pas le travail) et la bravoure (il n'a jamais peur et ne fuit pas la guerre). Par contre, comme le disent Pollet et Winter dans leur étude sur la société soninké, "le nyakamala ne pouvait se battre à la guerre; l'honneur et la gloire étaient hors de son atteinte. Il est tenu à l'écart de la chefferie de village." (Pollet \& Winter 1971 : 206). De par ses attributs, tout hàron est aîné des nyamakalaw (hommes de caste inférieure). Il est leur jatigi, étymologiquement l'hôte qui accueille l'étranger; ce qui implique un rapport symbolique de plus ancien à moins ancien sur le terroir. Et eu égard à l'importance de l'âge, le choix sémantique n'est pas fortuit. En effet, le pouvoir, l'administration du village revient aux hàronw et particulièrement à l'homme le plus âgé du lignage le plus ancien. Néanmoins, dans les instances de décision, les nyamakalaw sont conviés et il y ont voix délibérative. Mais l'intégration de ces castes inférieures est beaucoup plus difficile chez les Touareg à cause de l'existence de l'esclavage. Les asservis sont pudiquement appelés serviteurs par les chercheurs et l'autorité administrative. Ici, les hommes de caste supérieure (imajeren ou imochar et inelesmen) ne travaillent pas de leurs mains. Ils sont, traditionnellement guerriers pour les premiers et marabout pour les seconds.

12 En transposant ici les notions de séniorité ouverte et fermée de Balandier (Balandier 1974 : 106-107), nous pouvons affirmer que le premier type de séniorité, celui fondé sur l'âge biologique, est une "séniorité ouverte, ou relative" en ce sens que l'accès y est libre par la promotion des générations. Par contre, celle fondée sur la division en caste est une séniorité absolue, fermée. On peut devenir chef de famille et même de village si l'on est membre du lignage fondateur du village alors qu'il est impossible de changer de caste.

Les pouvoirs traditionnels au village

13 Ils sont exercés par les aînés tant sur le plan politique que religieux. Dans certains groupes ethniques il existe le chef du terroir (dugukolotigi chez les Bambara et kulofolo chez les Sénoufo) et le chef de village, administrateur des hommes (dugutigi chez les Bambara et kanfolo chez les Sénoufo). Selon les contingences historiques, ces deux fonctions peuvent être cumulées par la même personne ou exercées séparément. Le chef peut nommer des chefs de quartier et leur déléguer une partie de ses pouvoirs administratifs. L'autorité des chefs de culte y est aussi importante. Mais ils travaillent en étroite collaboration avec le chef de village et c'est d'ailleurs celui-ci qui, traditionnellement, autorise la pratique de tout culte dans son village. Dans les sociétés divisées en castes, chaque caste inférieure a généralement un chef. Il porte le nom de kuntigi chez les Bambara et les Malinké (jalikuntigi, numukuntigi, chef des jali, des forgerons). L'association des jeunes ainsi que celle des femmes existent et ont chacune leur chef.

Dans le domaine de la parenté, l'autorité est exercée par le chef de famille. Les chefs de lignage selon les localités peuvent bénéficier d'un pouvoir plus ou moins important. Celui-ci porte généralement sur le contrôle des relations matrimoniales et des ressources naturelles. Chez les Bambara et les Malinké, il arrive aussi que les chefs d'un 
certain nombre de villages aient en commun un ancêtre éponyme réel ou supposé. Ces villages forment alors une entité prenant la dénomination de kafo. Et généralement, c'est le plus âgé des chefs de village qui devient le kafotigii (chef de kafo). Pareille situation se retrouve aussi en pays dogon où l'administration recense certaines entités comme des villages alors qu'elles ne constituent que des quartiers de village-mère pour la tradition. C'est le cas, entre autre, de Sangha et de Ningari autour desquels gravitent, respectivement 18 et 14 villages.

Quels genres de relation les structures ci-dessus mentionnées entretiennent-elles avec le pouvoir?

Structures sociales traditionnelles et pouvoir

Il existe des ayants-droit des principaux pouvoirs traditionnels. Ainsi, pour ce qui concerne l'ensemble du village, il faut être l'homme le plus âgé du lignage fondateur du village. Et dans la plupart des groupes ethniques, la responsable des femmes est choisie dans la caste des hòronw. Il en est de même chez les jeunes. C'est pourquoi les premières associations des producteurs de coton (AV : association villageoise) étaient dirigées par les lignages des chefs de village. Le chef de village lui même en était le président, un de ses cousins ou frères cadets le trésorier et un de ses neveux ou fils lettrés le secrétaire. Ces postes étant les plus importants, les autres membres de l'association et même du bureau faisaient en fait office de figurants. La commission de contrôle était fictive et les fonds étaient à la disposition du chef. Ces bureaux, une fois mis sur pied, n'étaient jamais renouvelés. Ce sont les incidences des événements de mars 1991 en zone rurale qui ont, en certains endroits, entrainé une remise en cause de cet état de fait. Et c'était souvent des frondes impulsées par des partis politiques dont le chef de village n'était pas membre. Le problème qui se pose ici est, au fond, celui de la distinction à faire entre le patrimonial lignager du chef et celui de l'ensemble de la collectivité ou la compatibilité entre le mode traditionnel de gestion et le fonctionnement actuel du patrimoine collectif ou associatif. Selon Bagayoko, "Les richesses que les princes thésaurisent sous forme de ressources publiques ou d'Etat (toda) distinctes des biens communs familiaux (foroba) tout en ayant le même mode de gestion, servent prioritairement au maintien des conditions de reproduction du pouvoir d'Etat incarné par le souverain" (Bagayoko 1987 : 103). Plus haut, il écrit "que tout revienne de droit au souverain, ou qu'il le prenne par force, le résultat est le même. Seul son libre arbitre est juge de la manière de faire pour satisfaire ses désirs" (Bagayoko 1987 : 102).

17 Ce faisant, nous pouvons dire que le comportement de certains chefs traditionnels et même "modernes" n'est qu'une transposition d'une perception et d'un mode de gestion traditionnel, la gestion patrimoniale du pouvoir et des biens publics, dans un contexte régi, en principe, par des normes et règles différentes.

18 Mais il arrive que les exigences des "partenaires au développement" faussent les données. En effet, ceux-ci peuvent exiger certaines aptitudes ou qualités (être alphabétisé, femme, femme alphabétisée...) pour l'exercice de certaines responsabilités. Quand les membres du lignage du chef ne répondent pas à ces critères le village est obligé de faire appel à d'autres lignages y compris ceux d'hommes de castes inférieures. Le cercle du pouvoir s'ouvre, s'élargit, alors, contre la volonté des dominants.

Certaines vicissitudes de l'histoire peuvent faire que des lignages s'imposent. Ils s'agit de ceux comptant des anciens combattants de l'armée coloniale, des lettrés, de nouveaux riches... Et comme le disent les Bambara, il faut alors "laisser leur part", c'est-à-dire tenir compte de leur présence, les ménager. Ce qui s'est ajouté de nouveau 
avec le pluralisme politique fut la levée de bouclier contre les bureaux des associations villageoises et souvent la création d'associations parallèles, c'est ce qui a été appelé à l'époque la crise des organisations paysannes.

Pour ce qui concerne l'administration du village et à la gestion des ressources naturelles, elles se font de manière collégiale par l'ensemble des lignages à travers leurs chefs. Il arrive même que des responsabilités particulières soient confiées à certains lignages comme dans le delta central du fleuve Niger où l'on retrouve le chef de village, le chef des terres, le maître d'eau, des pâturages... Il s'en suit que, partout, le conseil du village et l'assemblée des chefs de famille jouent un rôle important. Les décisions se prennent dans le vestibule du chef de village, sous l'arbre à palabre ou sur la place publique (jè̀rè en bambara). Les chefs des lignages nyamakala y participent avec voix délibérative comme ci-dessus mentionné, mais, les jeunes et les femmes n'y sont pas conviés. En se plaçant dans le contexte des libertés individuelles on peut dire que chaque aîné a une délégation de pouvoir, une procuration lui permettant de parler et d'agir au nom de ses cadets. C'est l'une des raisons pour lesquelles, à l'époque du monolithisme politique (US-RDA, UDPM), un seul chef de famille pouvait voter en lieu et place de tous ses dépendants (10 à 100 personnes et même plus).

On peut donc conclure que les castes inférieures sont, la plupart du temps, exclues des postes de responsabilité et particulièrement du premier poste de direction; les jeunes et les femmes, eux, exclus de l'administration et de la gestion directe des ressources naturelles.

Il a été, ci-dessus, question de séniorité relative ou ouverte pour celle fondée sur l'âge et de séniorité absolue ou fermée pour celle fondée sur la division en caste. Mais n'existe-t-il pas un mécanisme d'intégration des castes inférieures en vue de la prévention de conflits pouvant surgir d'une telle situation?

23 Les castes inférieures sont intégrées dans le système par le phénomène du clientélisme. Traditionnellement, autour de chaque lignage hòron gravitent des castes inférieures. Celles-ci bénéficient de vivres (céréales) et occasionnellement de présents. En contrepartie, elles fournissent au jatigi (hôte), selon leur corporation, les instruments de travail dont celui-ci a besoin, organisent et animent ses cérémonies et manifestations et servent pour lui d'intermédiaires et d'entremetteurs. Enfin, un des aspects non moins importants est que les castes inférieures constituent des alliés pour leur jatigi dans la communauté. Et la valeur sociale d'un lignage hòron est proportionnelle à l'importance de sa clientèle. C'est ce que confirme un des informateurs de Bagayoko: "Celui qui bénéficie des libéralités d'un souverain sera comptabilisé parmi ses gens. Ce dernier ne pourra plus s'en détacher comme bon lui semble" (Bagayoko 1987 : 104).

24 Mais ce qui nous intéresse ici est si les nyamakala vont continuer à être des clients ou des citoyens à part entière dans le cadre de la décentralisation. Mieux, ne constate-t-on pas déjà une évolution dans ce domaine ainsi que dans celui des rapports fondés sur les critères d'âge?

Séniorité et dynamique sociale

Il a été ci-dessus mentionné qu'un certain nombre de villages peuvent revendiquer une ascendance commune. Mais à l'origine, ce sont des cadets qui se sont installés dans des hameaux pour échapper au pouvoir des aînés. A présent, la même méthode est toujours utilisée pour s'émanciper. Elle s'accompagne actuellement de l'entreprise de culture de rente (le coton) chez les Minyanka. L'exode rural est aussi un des moyens utilisés par 
les cadets pour échapper à l'emprise des aînés. Mais ces entreprises n'ont pas pour objectif une remise en cause du système mais visent plutôt une promotion individuelle, une volonté de rentrer dans le cercle du pouvoir, à défaut d'échapper, dans l'immédiat, à son emprise.

Les différentes castes, avec la monétarisation des rapports sociaux et la remise en cause de la traditionnelle division sociale du travail par la colonisation, ont vu l'horizon des professions s'élargir. Certains hommes de caste inférieure ont pu, ainsi, entreprendre des activités qui leur ont permis de se hisser au sommet de l'échelle sociale. Et cela de par les revenus qu'elles leur procurent ou de par la considération sociale liée à leur exercice.

Et comme ci-dessus mentionné, dans le cadre des rapports du village avec les "partenaires au développement", l'exercice de certaines activités ou fonctions nouvelles, mais non moins valorisantes, peut être conditionnée par l'alphabétisation ou par l'appartenance à une catégorie de sexe. C'est ainsi que des hommes de caste inférieure, des femmes et des cadets ont pu occuper des fonctions sociales qui les ont introduit dans le cercle du pouvoir. Tel est le cas de l'agent chargé de l'Etat civil à Zégoua et aussi des cas des présidentes des femmes du "projet moulin" au Mali (Béridogo 1997 : 57).

28 En conclusion, le cercle du pouvoir est théoriquement fermé. Mais les mutations sociales y introduisent des "intrus" par effraction. Peut-on transposer ce cercle dans un contexte démocratique? Les pouvoirs traditionnels et ceux des urnes sont-ils compatibles?

Pouvoir traditionnel et pouvoir des urnes (démocratie)

En se plaçant dans la logique de la démocratie occidentale, le pouvoir traditionnel est de nature réactionnaire, antidémocratique. Les hommes n'y sont pas jugés selon leur mérite mais selon leur naissance, leur origine. On naît hòron, nyamakala, femme... et on le demeure à vie, jouissant des prérogatives et des désavantages y afférents ; alors que les idéaux de mars 1991, dont la décentralisation s'inspire, s'inscrivent eux aussi dans l'optique du combat mené par la bourgeoisie occidentale depuis la Renaissance ; se faire juger sur la base de son mérite et non de son origine. Et même s'il y a des inégalités sociales criantes en occident, même si des "lignages" font main basse sur certaines circonscriptions électorales, il n'y est pas explicitement dit que les hommes naissent et demeurent inégaux. C'est ce qui fait écrire par Fougeyrollas que: "pour détruire le mode de production féodal et pour assurer son emprise sur le monde, la bourgeoisie du XVIIème siècle a besoin de dissoudre les anciennes "différences" justifiées par les préjugés nobiliaires et de poser en principes l'identité, l'égalité et l'universalité d'une nature humaine à partir desquelles seront seulement légitimes les différences résultant de l'activité des individus et des mérites acquis dans cette activité" (Fougeyrollas 1980 : 17-18).

30 Ainsi, dans le même sens au Mali, le mouvement démocratique, c'est-à-dire les associations qui luttaient pour la démocratie et la cause du pluralisme politique, a d'abord demandé, dans une lettre ouverte, au général Moussa Traoré d'accorder au peuple la possibilité "de choisir, en toute liberté, le parti politique qui lui paraitt le plus apte à défendre ses droits légitimes ${ }^{2 "}$. Suite au refus réservé à cette doléance, les deux associations politiques, le Comité National d'Initiative Démocratique (CNID) et l'Association pour la Démocratie au Mali (ADEMA), vont inscrire dans leur programme d'action la lutte pour l'instauration du pluralisme politique, et des libertés collectives 
et individuelles ${ }^{3}$. La cause une fois gagnée, la constitution stipulera et insistera plus que par le passé en son article 2 que : "Tous les maliens naissent et demeurent libres et égaux en droit et en devoir. Toute discrimination fondée sur l'origine sociale, la couleur, la langue, la race, le sexe, la religion et l'opinion politique est prohibées ${ }^{4}$.

Mais l'inégalité est une donnée importante des représentations sociales dans tous les groupes ethniques. C'est une situation de fait intériorisée par les uns et les autres. C'est pourquoi les Bambara affirment que Dieu a procédé à la création mais pas à l'égalité dans la société. "N'gala yé dani kè, $n$ 'ga a ma kélen ya kè". La liberté individuelle et le droit à la différence n'y sont pas, aussi, reconnus. Ainsi, lors des élections législatives du 13 avril 1997, suite aux difficultés d'obtention des cartes d'électeurs, la commission électorale nationale indépendante (CENI) autorisa les électeurs à voter avec les carnets de famille. Mais dans certaines localités comme à Lofigué dans le cercle de Kadiolo, les chefs de famille ont refusé les carnets aux cadets qui avaient opté pour les partis de leur choix. Presque partout, il n'est pas rare de voir les aînés confisquer les cartes d'électeurs des dépendants manifestant des velléités d'indépendance surtout quand les rapports entre partis politiques prennent une allure conflictuelle.

La décentralisation va-t-elle entraîner une mobilité sociale en zone rurale ? Le village va-t-il enfreindre l'ordre social traditionnel en faisant table rase des critères d'âge, de sexe et de caste, en jouant le jeu des urnes, de la démocratie et en accordant à chaque homme une voix, en permettant ainsi à la voix du cadet d'être différente et en même temps égale à celle de l'aîné au moment du vote et partant du décompte?

33 Pour l'instant ce n'est pas cette tendance qui se dégage. En effet, dans la phase du découpage, c'est-à-dire la constitution des communes, les villages devaient, librement, s'associer pour former les nouvelles entités. Le choix devait être fait par l'ensemble du village. Mais dans presque toutes les localités, les jeunes disent n'avoir pas été consultés ni invités à participer aux débats. Certains, comme ce cadet à Kadiolo, admettent la situation, estimant qu'en fait c'est l'affaire des aînés, les chefs de village et de famille.

Dans la commune rurale de Djidian, dans la circonscription administrative de Kita en pays malinké, les étrangers, c'est-à-dire tous ceux arrivés de fraîche date, ont été sevrés de parole. Dans celle de Zégoua, à l'extrême sud du Mali, en pays sénoufo, les autochtones ont juré de tout mettre en œuvre pour qu'un allochtone ne soit pas maire. Dans cette perspective, ils ont déjà commencé à les exclure de la gestion des infrastructures et institutions les plus importantes ou susceptibles de générer des fonds, borne fontaine publique, centre de santé communautaire, pharmacie communautaire... Aucun allochtone ne siège au conseil de village.

Le message des initiateurs du processus de décentralisation est-il parvenu intégralement et correctement aux bénéficiaires? Qu'est-ce qui a été entrepris pour créer une harmonie entre la constitution et les structures et pouvoir traditionnels ?

La politique nationale de communicationLes structures de communication

Pour rendre effective la décentralisation, une Mission de décentralisation (MDD) a été créée pour concevoir un travail institutionnel et organisationnel.

L'œuvre de décentralisation a ainsi commencé au niveau régional par un système de formation en cascade. Elle a d'abord ignoré l'administration. La Mission de décentralisation a formé dans chaque région un certain nombre de personnes en vue de la constitution des GREM (Groupe régional d'étude et de mobilisation). Ce GREM est une 
structure informelle sans statut ni règlement intérieur. Selon un des responsables, il a été voulu comme tel, avec un caractère léger souple et rapide. Ses membres résident en ville, c'est-à-dire dans les chefs-lieux de région et de cercle. Il s'agissait d'identifier et de choisir au niveau de ces deux types de localité les leaders d'opinion, les meneurs, les "grandes gueules" comme le disait un président de GREM, afin d'avoir avec soi tous ceux susceptibles de bloquer le processus. Tous ceux retenus au niveau régional ont subi, ultérieurement, une formation au chef-lieu de région et de retour dans leurs cercles respectifs, ils y ont formé une trentaine de personnes, dont des responsables politiques, d'associations villageoises, d'associations féminines... en provenance de tous les arrondissements. Au terme de leur formation ces différents auditeurs ont constitué le GLEM (Groupe local d'étude et de mobilisation) au niveau du cercle. Dans les arrondissements, ils devaient former d'autres personnes en vue de la constitution des GAEM, (Groupe d'arrondissements d'étude et de mobilisation). Ces GAEM devaient sillonner tous les villages en vue de la constitution des GM (Groupe villageois de mobilisation).

Mais les GAEM et GM n'ont pu être mis sur pied. Il a été demandé au Groupe Local d'Etude et de Mobilisation de surseoir au travail en vue de l'initiation d'un processus d'association de l'Administration.

Selon la Cellule de coordination régionale de Sikasso, la mise en train ayant été faite, on pouvait alors associer l'administration. Mais selon cette dernière, suite à l'échec des structures indépendantes et au blocage qui s'en est suivi, la Mission de décentralisation a réalisé que l'administration était incontournable et qu'il fallait lui faire appel.

C'est en fait à partir de janvier 1995 que l'administration a été associée au processus de décentralisation. Suite à cette association, un nouvel organigramme a été conçu. La Cellule de coordination régionale, au niveau régional, la Commission de découpage au niveau des cercles et les Equipes d'arrondissement au niveau des arrondissements.

Comment ces structures ont-elles véhiculé le message sur le terrain?

Les problèmes de communication

Le message des initiateurs du processus de décentralisation ne semble pas être parvenu correctement aux populations. L'information a été partielle et partiale et n'a pas atteint toutes les couches sociales.

Ces propos, recueillis lors d'une enquête de terrain par le Groupe de recherche sur l'Etat, la décentralisation et le foncier (GREDEF), confirment les difficultés de communication. C'est ainsi qu'un long silence fut la première réponse à la question "quelle est votre compréhension de la décentralisation". Doutant de la clarté de leur propos les chercheurs revinrent à l'attaque: "Est-ce que vous avez tous compris la question ?" Le chef de village répondit alors que "tout le monde a compris sauf moi qui suis sourd". Mais un de ses administrés le rassura: "Chef de village, à moins qu'il ne s'agisse d'une autre question ; sinon, celle là, même si tu l'avais entendue, tu n'y aurais rien compris. Même nous qui entendons correctement, elle n'est pas à notre portée".

Les populations demeurent donc généralement sous-informées. L'information demeure au niveau des chefs de village ou des délégués servant d'intermédiaires entre le village et ceux impliqués dans le processus de décentralisation. Beaucoup de villageois ont vaguement entendu parler du phénomène mais ignorent son contenu exact. Et l'indifférence est telle qu'ils ne cherchent pas à en savoir plus. C'est ce qu'illustre ces propos d'un villageois de Loutana : "C'est vous intellectuels qui avez monté toute cette 
histoire et nous attendons que vous veniez nous donner des réponses définitives au lieu de venir nous indisposer avec des réunions interminables." Les personnes qui disposent d'une bribe d'information ont de sérieuses appréhensions : "On dit que c'est le retour $\mathrm{du}$ pouvoir dans le terroir ; mais l'Etat dispose de gardes et n'arrive pas à récupérer les impôts ; comment est-ce que nous qui n'avons rien allons faire pour y arriver ?" disait l'un des villageois de Tinkolé.

Un certain nombre de faits peuvent expliquer cet état de sous et non-information.

1. Le scepticisme des "élites intellectuelles". Celles-ci doutent de la capacité des populations analphabètes ou non scolarisées à prendre leur destin en main. Elles les considèrent comme d'éternels mineurs taillables et corvéables à merci. Ces intellectuels ne se sont, donc, pas investis pour d'abord comprendre eux-mêmes le processus et ensuite transmettre le message à ceux qui n'ont pas accès à récriture et à la langue "officielle" qui est le français.

2. La réticence de certains administrateurs : Certains d'entre eux, en effet, craignent de perdre leurs privilèges avec la mise en œuvre de la décentralisation. C'est pourquoi un chef d'arrondissement la qualifiait de "très mauvaise réforme qui ne contribuera qu'à accentuer l'incivisme et la perte d'autorité dont souffre l'Etat depuis l'avènement du pluralisme politique". Et tous ceux qui s'inscrivent dans cette logique ne se sont pas suffisamment impliqués dans la mise en route du processus quand ils n'ont pas œuvré à son blocage.

3. Le choix des communicateurs locaux: Si "l'élite intellectuelle", dans son ensemble, doute de la réussite de la décentralisation, ceux choisis pour véhiculer le message auprès des masses rurales ont souvent vu en cette réforme une occasion de promotion individuelle. En effet, ce sont les lettrés en français, originaires des communes rurales, qui ont la plupart du temps été choisis pour transmettre le message à leurs parents. Le choix s'est porté sur les plus disponibles (retraités, partants volontaires à la retraite, compressés des sociétés et entreprises d'Etat, jeunes diplômés sans emploi...). Naturellement, cette couche de lettrés désœuvrés a pensé que c'était à elle que devait revenir la fonction de maire avec toutes les prérogatives jusque là réservées aux administrateurs qu'ils allaient remplacer. La masse étant analphabète, vivant en conséquence dans l'obscurité comme on le dit au Mali (kalan baliya ye dibi yé: l'analphabétisme c'est de l'obscurité), elles doivent donc être guidées à l'éclairage de ces quelques "lampes". Ils ont donc souvent transmis le message en fonction de leurs intérêts, provoquant des regroupements ou des scissions selon que ça les arrangeait.

Des ONG ont élaboré des modèles de communications et sont à l'œuvre sur le terrain. Le problème est qu'elles ne couvrent qu'une infime partie du territoire.

En dehors de cet état de sous et non-information, difficultés imputables en partie à la perception et aux attitudes des élites intellectuelles, d'autres existent et sont liées aux difficultés de traduction des concepts du français aux langues nationales et des applications ou usages particuliers découlant de la mauvaise interprétation de ces concepts.

51 Ainsi, au niveau des concepteurs et exécutants du processus de décentralisation, certains concepts prêtent à confusion. Il y a un problème de différenciation entre démocratie et décentralisation. La démocratie, elle, a d'abord, été traduite par foroba fanga, le pouvoir de tout le monde. Mais il a été estimé que cette formulation avait une connotation anarchique, renvoyant à l'idée que le pouvoir appartenait à tout le monde 
et en même temps à personne. Alors bèjè fanga (le pouvoir avec lequel tout le monde est d'accord) fut préféré. C'est le concept de décentralisation qui pose le plus de problème et qui jusqu'ici n'a pas eu de traduction définitive.

En bambara et en malinké, deux notions reviennent le plus souvent, celles de mara et defanga. Mara renvoie à l'exercice de l'autorité à tous les niveaux de la société, y compris au sein des lignages: rapports aîné/cadet, famille, village, kafo, pouvoir central... Le terme de fanga. quant à lui renvoie à la notion de force, à l'exercice du pouvoir en dehors du lignage, au niveau le plus élevé, soit le pouvoir central. Le concept de décentralisation est donc traduit par mara ka ségi so (le retour de l'autorité, du pouvoir à la maison, au terroir), soit un pouvoir dont la base, le terroir avait été exproprié et qui lui est restitué. Les expressions de mara kanan bo (restaurer le pouvoir, procéder à sa réfection) de mara ka cèbo ou ka fanga cè bo (élargir le cercle de participation au pouvoir) sont aussi utilisées.

$\mathrm{Au}$ nord du pays sénoufo, l'expression de wu ya yo (prenons-nous en charge ou acquérons notre indépendance) est utilisée. A l'extrême sud, on parle de wa bi wu ya ma (travaillons pour nous-mêmes, procédons à l'auto-développement).

Perceptions et pratiques sur le terrain

Souvent, dans la mise en pratique des concepts, c'est la confusion totale. Certains lignages qui ont perdu le pouvoir estiment que la réforme a été entreprise pour eux. Des villages qui avaient servis de chef-lieu pour d'anciens pouvoirs (chef-lieu de canton, d'entité politique précoloniale) ou ayant été fondés avant les autres ont estimé que de facto, ils devaient servir de chef-lieu de commune. Chacun interprète les concepts à son avantage. La décentralisation est perçue comme la réinstauration des pouvoirs traditionnels précoloniaux ou coloniaux dans tous leurs aspects y compris ceux qui vont à l'encontre de la constitution qui sert de support à la dite reforme. C'est ainsi que les pratiques inégalitaires consistant à exclure du pouvoir ou des instances de direction les étrangers, les hommes de caste inférieure, les jeunes, les femmes... sont souvent reconduites.

Même au niveau des concepteurs (l'Etat et ses intellectuels) on semble oublier que beaucoup de chefs de canton, et partant leur village comme chef-lieu de canton, ont été choisis par le colonisateur en reconnaissance de leur allégeance à l'administration coloniale. La division des colonies en cantons relève du type d'organisation adopté par la colonisation pour son administration. Ces cantons ne sont nullement des structures traditionnelles. Leurs limites ainsi que leurs dénominations relèvent très souvent de l'arbitraire.

Même si les textes de création mettent en avant les dimensions de libertés individuelles et collectives, les acteurs, eux, avancent prudemment sur le terrain. Ils évitent les remises en cause explicites des aspects antidémocratiques du pouvoir traditionnel. Les "fondamentalistes culturels", eux, prônent le retour à un passé présenté comme l'exemple achevé de démocratie, de décentralisation. Alors que, comme ci-dessus mentionné, même s'il y a eu déconcentration du pouvoir, voire décentralisation dans la période pré-coloniale, ce n'était pas dans un contexte de pluralisme politique, de compétition ouverte aux uns et aux autres sans discrimination d'âge, de sexe ou d'origine sociale. Un effort est cependant entrepris par certaines associations de femme, des ONG et même la Mission de décentralisation (l'organisme de mise en œuvre du processus de décentralisation) pour la prise en compte des droits de la femme. Le 
discours relatif à la cause des castes inférieures et des jeunes est très timide voire inexistant.

Conclusion et perspectives (ou plutôt conjectures)

57 Le découpage, la constitution des communes ont été faits sans l'association des femmes et des jeunes et souvent des étrangers. Les élections communales ont été reportées. Le choix des conseillers municipaux et du maire va-t-il encore les exclure, ou le cercle de participation au pouvoir va-t-il s'élargir pour faire place aux marginalisés?

Pour l'instant nous ne pouvons que conjecturer à partir des tendances qui se dégagent et de l'expérience des élections précédentes.

Contrairement à l'ancien code électoral, l'ordonnance n 91-074/P-CTSP. du 10 octobre 1991, le nouveau, la loi nº 97-008 du 14 janvier 1997, permet de s'organiser et de concourir en dehors des partis politiques enregistrés par l'autorité administrative et cela sur la base de candidatures ou de listes indépendantes. Les villages, de manière consensuelle, peuvent élaborer et présenter des listes indépendantes d'où seraient exclus les étrangers. Cela supposerait qu'ils se mettent au-dessus ou à côté des partis politiques, qu'ils surmontent les contradictions internes, rivalités intra et extra-lignagères... Mais si le pluralisme politique est effectif dans la commune, le consensus sera difficile voire impossible, chacun se battant pour la liste présentée par son parti politique. La compétition aidant, on peut alors recourir et même faire figurer sur sa liste tel allochtone pour bénéficier de son soutien matériel ou moral (moyen financier, savoir et savoir-faire, audience dans tel milieu...).

Pour ce qui concerne le rapport des jeunes et des femmes au pouvoir, tous les aînés, qu'ils soient autochtones ou allochtones, de caste supérieure ou inférieure sont d'accord sur le fait que "le pouvoir n'est pas une affaire de jeune ni de femme" : il revient aux aînés. Ce schéma a toutes les chances d'être mis en pratique lors des prochaines élections si les populations ne se mettent pas en marge du processus comme elles l'on fait jusqu'ici.

61 Mais ultérieurement, c'est une prise de conscience, la manifestation d'une conscience de groupe donnant naissance à des groupes de pression qui peut initier une véritable évolution. En ce moment, ils peuvent jouer sur les rivalités entre partis politiques et exiger la prise en compte de leurs préoccupations en échange de leurs voix ou mieux exiger le partage du pouvoir, soit leur présence sur les listes pour résoudre eux-mêmes leurs problèmes (Nadio $1997: 60$ ).

\section{BIBLIOGRAPHIE}

Abeles. M. \& C. Collard (dir.) 1985. Age. pouvoir et société en Afrique noire. Paris : Karthala.

Bagayoko, S. 1987. "L'Etat au Mali, Représentation, autonomie et mode de fonctionnement". In : Terray E.(dir.) L 'Etat contemporain en Afrique Paris : L'Harmattan : 91-122.

Balandier, G. 1974. Anthropo-logiques. Paris : PUF. 
Béridogo, B. 1997. "Femmes rurales et innovations techniques et technologiques au Mali". Bulletin de l'APAD 13 : 51-61.

Félix, J. 1996. "L'élaboration de la politique de décentralisation au Mali : des logiques plurielles", Bulletin de l'APAD $11: 148-163$.

Fougeyrollas, P. 1980. Sciences sociales et marxisme. Paris : Payot.

Les Echos. n³9 du 17 au 31 août 1990, supplément. Bamako : édition Jamana.

Les Echos. $\mathrm{n}^{\circ} 45$ du 26 octobre au 9 novembre 1990. Bamako : édition Jamana.

Meillassoux, C. 1975. Femmes, greniers et capitaux. Paris : Maspéro.

Nadio, A. 1997. Femme et enjeux de pouvoir dans le cadre de la décentralisation. Bamako : ENS, DER PPP (mémoire de fin d'études).

Pollet, E. \& G. Winter 1971. La société soninké. Bruxelles : Institut de sociologie.

Sindzingre, N. 1985. "Aspects de l'aînesse sociale dans les communautés villageoises fodonon, (Senufo de Côte d'Ivoire)". In : Abeles M.\& C. Collard C (dir.) Age, pouvoir et société en Afrique noire, Paris : Karthala : 149-170.

Soumaoro, L. 1997. Décentralisation et enjeux de pouvoir traditionnel dans la commune rurale de Zégoua. Bamako : ENS, DER PPP (mémoire de fin d'études).

Suret Canale, J. 1977 (2e éd.). L'Afrique noire (tome 2 : l'ère coloniale 1900-1945). Paris : Éditions Sociales.

Constitution du 25 février 1992 de la République du Mali. Bamako : Secrétariat général du gouvernement.

Lois et Décrets de la décentralisation 1996. Bamako : Primature-Mission de décentralisation.

Loi n97-008 du 14 janvier 1997 portant "Loi électorale". Bamako : Secrétariat général du gouvernement.

Ordonnance $\mathrm{n}^{\circ}$ 91-074/P-CTSP du 10 octobre 1991 portant "Code électoral en République du Mali", Bamako, Secrétariat général du gouvernement.

\section{NOTES}

1.Nous n'utilisons pas la notion de caste dans l'assertion malienne du terme pour laquelle caste se traduit par nyamakala et Mron par noble. Nous estimons que c'est toute la société qui est divisée en caste. Comme Pollet E. et Winter G., "en rompant avec l'usage établi, nous conformons la théorie de la caste ouest-africaine au schéma classique de cette institution, qui est celui d'un système englobant tous les membres de la communauté, condition rappelée récemment par L. Dumont 1966 : 272" (Pollet E. et Winter G. 10971 : 206).

2.Journal Les Echos. n 39 du 17 au 31 août 1990, supplément, Bamako, édition Jamana. 3.Journal Les Echos, $n^{\circ} 45$ du 26 octobre au 9 novembre 1990, Bamako, édition Jamana. 4.Constitution du 2 février 1992 de la République du Mali, titre premier, article 2. 


\section{AUTEUR}

BRÉHIMA BÉRIDOGO

Université du Mali - FLASH, BP 241, Bamako (Mali). Tél. : 223/22 2189. Fax. : 223/22 75 88. 